\title{
IMPROVED INVESTMENT PERFORMANCE USING THE PORTFOLIO DIVERSIFICATION INDEX
}

\author{
Francois van Dyk* \\ University of South Africa \\ vdykf@unisa.ac.za
}

\author{
Gary van Vuuren+ \\ North West University \\ gary.vanvuuren@nwu.ac.za
}

Paul Styger\#

North West University

paul.styger@nwu.ac.za

July 2011

\begin{abstract}
The residual variance method is the traditional method for measuring portfolio diversification relative to a market index. Problems arise, however, when the market index itself is not appropriately diversified. A diversification measurement (Portfolio Diversification Index), free from market index influences, has been recently introduced. This article explores whether this index is a robust and 'good' diversification measure compared with the residual variance method. South African unit trusts are diversification-ranked using the two measures and the results compared to the ranking results of several risk performance measures. Measuring relative concentration levels allows concentration risk to be effectively managed, thereby filling a gap in the Basel accords (which omit concentration risk).
\end{abstract}

\section{Keywords}

Diversification, concentration, residual variance, Omega ratio, Portfolio Diversification Index

\footnotetext{
*Mr Francois van Dyk is a senior lecturer in the Department of Finance, Risk Management and Banking, UNISA, Pretoria, South Africa.

+ Dr Gary van Vuuren is an extraordinary professor at the School of Economics, North West University, Potchefstroom, South Africa.

\#Prof Paul Styger is a programme leader in Risk Management at the School of Economics, North West University, Potchefstroom, South Africa.
} 


\section{INTRODUCTION}

As modern financial markets become larger, more highly developed, more transparent and more efficient and with market participants gaining access to ever-increasing data, the tools and analysis employed will (and will have to) develop in tandem. With the markets becoming ever more efficient, the skill of attaining above-average risk-adjusted returns will become ever more difficult. The focus of investors and market participants should be prompted to shift towards effective portfolio diversification in order to help ease the task of attaining above-average risk-adjusted returns.

Diversification is a major component of portfolio management and a core objective for combining assets in the construction of portfolios, while also being woefully misunderstood and complex to model mathematically. To date, the Basel Committee for Banking Supervision (the $B C B S$ ) has not included concentration risk in the Basel regulatory capital accords. A proposed treatment of concentration risk was included in the Basel II proposal documents in early 2001, but due to its mathematical complexity and difficulties of implementation, it was subsequently excluded. This research presents a novel methodology to measure relative concentration levels and hence effectively manage concentration risk, thereby bridging a gap in both the risk literature and the practice of concentration risk mitigation.

Even though diversification is fundamental to portfolio construction, the most common and traditional manner in which to measure the degree of portfolio diversification remains inexact. The 'traditional' diversification method (the residual variance (RV) method) correlates the returns of the portfolio with the returns of, for example, an index. Though this method is easily understood and not exceptionally time consuming, it does have some shortcomings (Gopi, Dugmore \& Bradfield, 2006). One aspect of this approach is its use of the market index as a benchmark for complete diversification. Problems thus occur when the market index itself is not appropriately diversified across industries (Gopi et al., 2006) and is therefore not representative.

One of the more sophisticated tools developed to cope with the expansion and evolvement of financial markets is the Portfolio Diversification Index (PDI), which is a measure of portfolio diversification. This new diversification measure also improves on the traditional approach of measuring diversification, as it is free of market index influence (Smith, 2006). The PDI first appeared in the academic literature in 2006 (Rudin \& Morgan, 2006).

The aims of this study are twofold. First, the goal is to establish whether or not the PDI is robust and reproducible (i.e. a good and/or useable and accurate diversification measure relative to the RV method, which is often used to determine diversification). Second, it aims to determine whether the PDI can be used as a tool by fund managers to assist in constructing funds (or changing the composition of existing funds) to reduce portfolio risk without reducing the portfolio return.

The PDI is free from the overall market index's influence, so this measure could improve on traditional approaches (which remain inexact) used to measure portfolio diversification. The PDI may also prove to be yet another tool, as it measures the degree of diversification that specific shares add to portfolios while also being able to quantify the diversification of a fund after fund adjustments (i.e. either fund composition or the weighting structure).

The limitations of this study include the following: 
1) research and literature regarding the PDI is very limited indeed - due to the relative novelty of the approach;

2) the hedge fund industry has not been highly regulated in the past (this is true globally, but is particularly true of South Africa), so the availability of historical data is severely limited; and

3) the availability of appropriate data e.g. investment funds, market indexes, etc. in terms of frequency and range, is limited in some cases.

Also the BCBS does not currently provide any formal and constructive guidance or framework concerning either concentration measures or portfolio diversification, and how such measures should be used or constructed.

The remainder of this paper is arranged as follows: section 2 provides a literature review of Modern Portfolio Theory as well as the elements of portfolio diversification. The construction of the PDI is discussed in section 3 , while section 4 focuses on the data and the processes performed thereon. Empirical results obtained are also analysed in this section. The results of the PDI as a diversification measure and the PDI as a fund management tool are presented in sections 5 and 6 respectively. Section 7 provides conclusions for the paper and section 8 suggests future research suggestions.

\section{LITERATURE SURVEY}

The objective of Modern Portfolio Theory is to ascertain a balance between risk and return by identifying the acceptable level of risk tolerance and then to find a portfolio with the maximum expected return for that specific risk level. Ultimately, Modern Portfolio Theory (MPT) proposes the manner rational investors will use diversification in order to achieve optimisation of their portfolio (Reilly \& Brown, 2006). Three primary elements exist within portfolio theory:

1) risk,

2) return and

3) diversification, while diversification also features as one of the three problems of portfolio construction along with asset allocation and timing (D'Ambrosio, 1976).

Since the amount of risk associated with a portfolio is dependent on the extent to which the assets in a given portfolio move together (covariance), the assumption can be made that the risk of a portfolio is not simply the weighted average risk of the assets within the portfolio. The covariance of a portfolio is reliant on two factors, namely:

- variability (standard deviation) of the individual assets, and

- the relationship (correlation) between different assets included in the portfolio.

Since the inception of portfolio design, diversification has been a central theme, with one of its core objectives being combining assets in the portfolio (Markowitz, 1952). The risk faced by investors can be altered by diversifying the portfolio - i.e. some of the risk present in the portfolio can be eliminated by holding different assets. By definition diversification is a portfolio strategy designed to reduce exposure to risk by combining different assets with low correlation of return (Reilly and Brown, 2006). A well-diversified portfolio is defined by Gopi et al., (2006:1) as "... one where the return, and consequently the risk, arises from as many unrelated (or independent) sources as possible". 
When investments are made at a certain time, no certainty exists regarding which investments will fail and which will succeed (Correia, Flynn, Uliana \& Wormald, 2003). It is thus sensible to diversify into a number of (different) investments with the expectation that the successful investment will at least compensate (the investor) for the investments that were not successful. Hattingh (2006) argues that diversification is the simplest (though not the most exact) form of hedging.

In a portfolio setting the process of diversification has the effect of reducing the variability of portfolio returns, according to Correia et al. (2003), while Hattingh (2006) states that when various assets with 'imperfect correlations' are included in a portfolio they will reduce the general level of risk of that particular portfolio.

Ultimately the diversification process serves to eliminate all unique or unsystematic risk from a portfolio, although the standard deviation (of a portfolio) will eventually reach the level of the market portfolio (where all unsystematic risk will be diversified away), but the systematic risk (market risk) will remain.

Investors accept and realise that diversification is a necessity, but usually give little attention to the amount of diversification necessary. How do investors know whether they are diversified, and thus how is portfolio diversification determined? This section will present only the RV method of measuring diversification: the construction of the PDI will be discussed later.

Traditionally, diversification is measured relative to a market index by regressing the portfolio returns against the market index (Gopi et al., 2006; Barnea \& Logue, 1973). This is arguably the most common and most widely used measure of diversification. The residual variance represents the extent of diversification still achievable in the particular portfolio. The smaller the residual variance, the more diversified the portfolio (Gopi et al., 2006). However, if the market itself is not well diversified, this traditional method used in practice provides an imperfect result. This tends to be even more so in highly concentrated markets, for example the South African market. The PDI, which is a quantitative measure of diversification, overcomes the problem of market influence.

A relatively new measure of diversification, the PDI, evaluates the diversification of a portfolio quantitatively and also overcomes the problem of market influence faced by other methods, specifically the 'traditional' RV method (Smith, 2006). This 'new' diversification measure, which is free from market influence or any market indices, was proposed by Rudin and Morgan (2006). It is important to note that the PDI is constructed using Principal Component Analysis (PCA), which quantifies the number of independent factors in a portfolio (Rencher, 1998). The diversification properties of a portfolio are then conveyed by means of the relative strength of these factors. The PDI enables diversification to be compared across different portfolios and/or over different time periods, while also ascertaining whether the addition of new securities improves diversification of a specific portfolio and by how much (marginal PDI) (Smith, 2006).

PCA is a mathematical procedure that transforms correlated variables into the same number of uncorrelated variables, referred to as principal components (Jolliffe, 1986). The first principal component accounts for as much of the variability in the data as possible, and each succeeding component accounts for as much of the remaining variability as possible (Rudin \& Morgan, 2006). It should be noted that the focus is not on the factors or variables themselves, but only on the makeup of their relative strength and how that strength delivers the diversification characteristics of a portfolio. PCA can be conducted on either covariance or correlation matrices, although Rudin and Morgan (2006) regarded a correlation matrix inappropriate for 
quantitative portfolio analysis because of its radical inefficiency. For a detailed review of PCA see Jolliffe (1986), Fung and Hsieh (1997a), Fung and Hsieh (1997b), Fund and Hsieh (2002) and Jolliffe (2002).

\section{PDI CONSTRUCTION}

The percentage of the total variance that each component has in the portfolio is calculated using equation (1), which asserts that the percentage of the total variance (relative strength) attributed to the $i^{\text {th }}$ component is given by (Rudin \& Morgan, 2006:87):

$$
W_{i}=\frac{\lambda_{i}}{\sum_{k=1}^{N} \lambda_{k}}
$$

where

$$
\begin{aligned}
& W_{i} \text { is the percentage contribution of factor i to total volatility } \\
& \lambda_{i} \text { are the eigenvalues as generated by PCA } \\
& N \text { is the number of securities or instruments } \\
& k \text { is the summation variable and the sum of the weights always sums to } 1 .
\end{aligned}
$$

The principal components of the portfolio and their relative strengths are evaluated by PCA when the historical returns of the portfolio composition are known. The vector of the components' relative strengths indicates the number of the portfolio's truly independent components as well as their relative strength (Smith, 2006). The vector provides complete information about the diversification magnitude of the portfolio. Rudin and Morgan (2006:82) define the PDI as in equation (2).

$$
P D I=2 \sum_{k=1}^{N} k W_{k}-1
$$

Where PDI is the centre of mass of the principal component's relative strength vector and

$N$ is the number of securities and

$k$ is the summation variable.

The PDI evaluates the effective truly independent components within a portfolio (Rudin \& Morgan, 2006), so the PDI is the centre of mass of the principal component's relative strength vector (Rudin \& Morgan, 2006), referred to as the centre of gravity or balancing point of the independent components (Gopi et al., 2006). The PDI thus measures the degree of 'frontloading' of the vector and ultimately summarises the properties regarding diversification into one statistic.

According to Jolliffe (2002) the principal components are uncorrelated and are ordered by declining variance. The contributions of the factors to the variability of the portfolio are ranked from 1 to $N$ in decreasing order. Furthermore, the magnitude of the eigenvalue is known as the height and represents the degree of its contribution to the volatility. FIGURE $\mathbf{I}$ indicates the balancing point of such a portfolio with the hypothetical case of equal factors. As a result of the equal factors the balancing point is as far over to the right as possible (Gopi et al., 2006). 


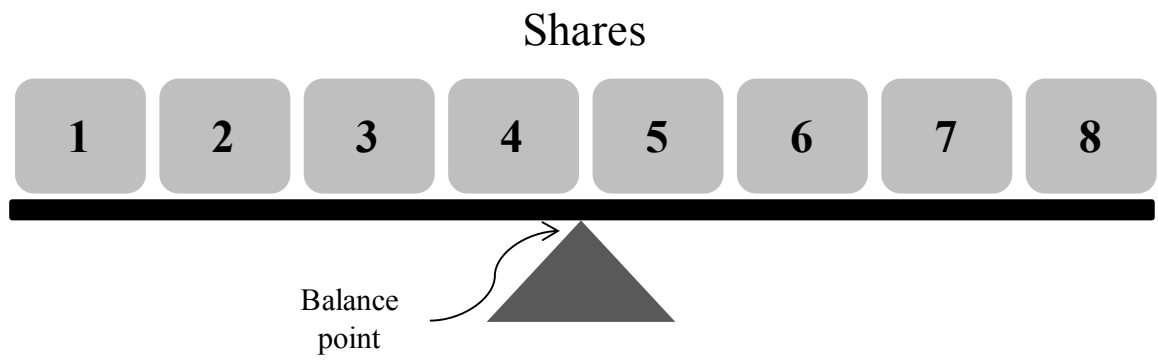

FIGURE 1 The maximum PDI in a hypothetical perfectly diversified portfolio

Source: Gopiet al. (2006:7)

Note that FIGURE 1 represents a diversification picture of an unrealistic hypothetical portfolio, while the numbered blocks in FIGURE 1 represent the components, for example shares, of this hypothetical portfolio. In a highly concentrated market such as the Johannesburg Securities Exchange (JSE) the volatility contribution of the large factors are likely to be far more than the contribution of the smaller factors (Smith, 2006). The first few factors would thus dominate, causing the balancing point to shift to the left as illustrated in FIGURE 2.

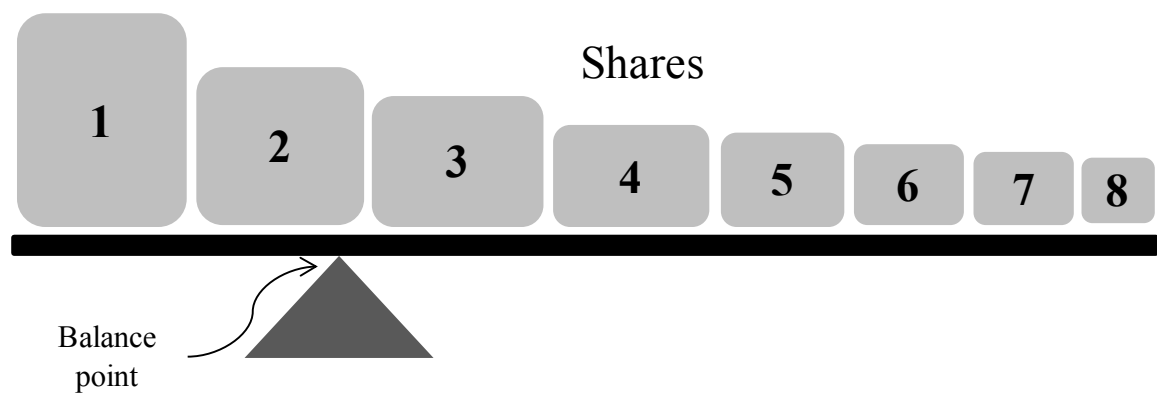

FIGURE 2 PDI in a hypothetical portfolio influenced by concentrated factors

Source: Gopiet al. (2006:7)

It can thus be concluded that a portfolio with a smaller PDI is the result of a less diversified portfolio.

The PDI has the following properties, according to Rudin and Morgan (2006:82):

- a portfolio which is dominated by a single factor and thus is completely undiversified, $\left(W_{1} \approx 1, W_{2} \leq k \leq N \approx 0\right)$, will have $\mathrm{PDI}=1$,

- a portfolio which is ideally (completely) diversified $\left(W_{k} \approx 1 / N\right)$ for all $k$, will have $\mathrm{PDI}=N$, where $N$ is the number of assets in the portfolio, and

- any positive change in front-loading (explanatory power moves from $W_{k}$ with higher to $W_{k}$ with lower $k$ ) reduces the index.

Although this falls outside the scope of this study, it is important to note that Rudin and Morgan (2006) propose two PDI approaches:

a) one for a portfolio and 
b) one for a collection of assets without considering any particular portfolio.

The PDI can also be used as a 'marginal' calculation, marginal portfolio diversification, to determine the degree of (additional) diversification gained (or lost) by adding shares to the portfolio. Marginal portfolio diversification can be defined as (Smith, 2006):

$$
\text { Marginal } \mathrm{PD}(k)=\mathrm{PDI}(\mathrm{Pool} \text { size }=k)-\mathrm{PDI}(\mathrm{Pool} \text { size }=K-1)
$$

where $K$ is the number of assets in the portfolio.

The portfolio risk will decrease as more assets or shares with low covariance (assets or shares that behave independently) are added to the portfolio (Smith, 2006; Statman, 1987). According to Stallman (2004), the higher the concentration of a particular fund the less diversified it will be; thus an inverse relationship exists between concentration and diversification.

In order to draw conclusions about the concentration of the funds, the Herfindahl-Hirschman Index ( $\mathrm{HHI}$ ) will be used as the concentration measure. The $\mathrm{HHI}$ is shown in equation (4) where

$$
\begin{aligned}
& W_{i}^{2} \text { represents the } i^{\text {th }} \text { shares investment weight (Smith, 2006:18): } \\
& H H I=\sum_{i=1}^{N} W_{i}^{2}
\end{aligned}
$$

Where $N$ is the number of assets and $W_{i}$ is the weight of the $i^{\text {th }}$ share in the portfolio.

For further detail on the HHI, see Hovenkamp (1993).

\section{DATA}

The empirical research was conducted using three randomly selected South African unit trusts of growth fund nature (Funds $A, B$ and $C$ ), which were selected from the entire South African unit trust growth fund sector. An additional two South African unit trusts were included in the research, and were deliberately selected to act as comparisons. The first of these two additional funds (Fund D) was the best performing growth fund in South Africa in terms of return for the preceding five years according to Equinox (1999), while the second of these funds (Fund $\varepsilon$ ) was the best overall performing South African unit trust for the preceding five years. It should be noted that Fund $\varepsilon$ is, however, not a growth fund but a fund of small cap nature. These funds were included in the research to compare how the three (randomly) selected funds match up against these funds in terms of diversification and performance. In addition, the average four year risk-free rate ( $8.12 \%$ ) was used as the risk-free rate, and all funds analysed were based on the equity component holding only, i.e. all cash and bonds were excluded. Lastly, all the shares contained in the funds were selected from shares listed on the Johannesburg Securities Exchange, while shares from all sectors could be included in the funds. The reasoning behind the use and inclusion of only five funds was that there are difficulties associated with procuring the relevant information, which is often proprietary and highly confidential.

The historical weekly closing prices of the shares comprising the five funds over the preceding four year period, 1 July 2003 to 30 June 2007, were obtained from McGregor BFA (stock market and fundamental research data provider), and hence the PDI was calculated using the historical four-year weekly returns for the period 1 July 2003 to 30 June 2007. The composition or component weights for each fund were also a key part of the PDI construction or calculation process. The weight of each share in each fund was taken at 31 March 2007, as this date was not only the most recent but also the most reliable (complete) date available. The composition of 
the five funds was sourced in electronic format from the Department of Financial Management at the University of Pretoria, as the Department publishes the Unit Trust Survey on a quarterly basis.

Proxies were used for any 'missing' or incomplete data, i.e. closing prices. This was due to the fact that some shares were not yet listed on the Johannesburg Securities Exchange (due to the time span of this empirical study) or that no closing prices were available. This problem was solved by backfilling these dates with a sector proxy. This proxy consists of the returns of the sector that the share (which required backfilling) hails from on the Johannesburg Securities Exchange. Thus if, for example, Anglo American had missing data, the returns from the platinum sector were used to fill these gaps.

A proxy was also used for Fund C for the period 1 July 2003 to 31 March 2004, as data for this period was not acquirable. The reason for the unavailability of data for this period is that Fund $C$ underwent a number of fund management administration alterations during this period. The proxy used for Fund C during this period was an average of Fund A and Fund B's weekly closing prices for the corresponding week. As from 1 April 2004 to 31 June 2007, the correct weekly closing prices were used for Fund $C$. The return performance of the funds is one area where this proxy might have played a role, as Fund $C$ performed phenomenally in especially the first year of this study (July 2003 to June 2004).

The used proxy was chosen to be as representative as possible in order to minimise any or potential bias that might be created. Furthermore, the use of an average of the other two primary funds (Fund $A$ and $B$, which are also of a growth fund nature) protects Fund C from having a widely different (starting) base compared to the other two primary funds.

The data required in order to calculate the PDI was:

- the current compositions of the five growth funds under investigation and

- the return history of all the shares held in the funds being investigated.

The procedure (in detail) to calculate the PDI is:

- the closing prices of the shares comprising each fund are accumulated, where after the data relating to shares that were not yet listed on the Johannesburg Securities Exchange (due to the time span of the study/analysis) or the shares having no returns are backfilled with returns of a proxy. In this case the proxy that is used is the return of the specific shares sector as explained above,

- a Principal Component Analysis (PCA), using SAS ${ }^{\circledR}$ (2009) (business analytics software), is then conducted on a co-variance matrix of these series,

- a series of uncorrelated factors (eigenvalues) that represent the volatility of the returns is the result of the PCA,

- order these factors from most to least significant and

- substitute these factors into the PDI formula (equation (2) as defined in Section 3).

The RV method of measuring diversification involves fitting a regression of portfolio returns against the returns of the market index. This Ordinary Least Squares (OLS) regression analysis using Eviews@ (2008) (econometric and statistical analysis software) is done from 1 July 2003 to 30 June 2007, which is the same time period used as for the PDI analysis, for comparative reasons. The weekly closing prices or returns for the funds being analysed were required, as were the weekly closing points or returns for the market index. The Johannesburg Securities Exchange 
All Share Index (ALSI) was taken as the market index for this study and only equity was included in the regression analysis.

The process or procedure to obtain the degree of fund diversification by using a regression analysis (traditional method) is as follows:

- the weekly returns of the five unit trusts must be calculated (this is done by using the weekly closing prices),

- the weekly returns of the market index have to be calculated (again the weekly closing points are used to obtain the weekly returns),

- the returns of each of the five unit trusts simply have to be regressed against the market index, in this case the Johannesburg Securities Exchange All Share Index (ALSI) and

- the regression results are then traditionally interpreted as the smaller the residual variance, the more diversified the portfolio (unit trust represents the portfolio in this study).

\section{RESULTS - PDI AS DIVERSIFICATION MEASURE}

\subsection{Method of testing}

In order to ascertain whether or not the PDI is a good diversification measure compared to the traditiona/RV method, the PDI is tested 'head-to-head' against the RV method. This is done by ranking the funds in accordance with the results of a number of different performance measures and also concentration. Although the two diversification measures and the performance measures or ratios are not directly comparable, as they measure different concepts, this concept of testing was adopted because there is no statistical method to do so. The idea is to ascertain whether or not the PDI is in line (or perhaps better aligned) with the results of the performance measures, than the RV method.

\subsection{PDI results}

TABLE 1 shows the results from the PDI calculation for each of the five unit trusts.

It is evident from the PDI results in TABLE 1 that Fund $D$ is the most diversified of the funds, while Fund $C$ is the least diversified. It is clear from the PDI results, presented in TABLE $\mathbf{1}$, that not one of the funds is completely diversified: for example, for Fund $D$ (which is the most diversified of the funds) to be completely diversified it should have a PDI = 38 and not 12.7 . 


\section{TABLE 1: PDI results}

\begin{tabular}{|c|c|c|}
\hline Fund & PDI & Number of shares in portfolio \\
\hline$A$ & 4.44 & 33 \\
\hline B & 7.68 & 50 \\
\hline C & 1.02 & 34 \\
\hline $\mathrm{D}$ & 12.70 & 38 \\
\hline$\varepsilon$ & 6.51 & 50 \\
\hline
\end{tabular}

Source: Statistical analysis

To recapitulate: Gopi et al. (2006:7) state that for a completely undiversified portfolio which is dominated by a single factor the $P D I=1$, but for a completely diversified portfolio the PDI = the number of assets in the portfolio, $N$. Thus, the smaller the PDI measure the less diversified the particular portfolio, while, conversely, a more diversified portfolio will have a larger PDI measure.

TABLE 2 presents the results of the two diversification measures side by side, with the best referring to the best or most diversified fund and the worst referring to the worst or least diversified fund. It should be remembered that for the RV method the extent of diversification still achievable in the portfolio will be represented by the variance of the residuals, and the smaller the RV, the more diversified the portfolio.

TABLE 2: PDI and traditional diversification measure results

\begin{tabular}{|c|c|c|c|c|}
\hline \multicolumn{2}{|c|}{ PDI } & & \multicolumn{2}{|c|}{$\begin{array}{c}\text { Traditional } \\
\text { Diversification Measure }\end{array}$} \\
\hline PDIScore & Fund & & Fund & Residual variance \\
\hline 12.70 & $D$ & Best & $\varepsilon$ & $4.61 \varepsilon-04$ \\
\hline 7.68 & B & & D & $4.90 \varepsilon-04$ \\
\hline 6.51 & $\varepsilon$ & & A & $5.55 \varepsilon-04$ \\
\hline 4.44 & A & & c & $3.16 \varepsilon-03$ \\
\hline 1.02 & C & Worst & B & $5.84 \varepsilon-02$ \\
\hline
\end{tabular}

\section{Source: Statistical analysis}

TABLE 2 shows that the two diversification measures are not in line with each other. According to the results presented in TABLE 2, the PDI measure states that Fund D is the best diversified fund among the funds included, while the traditional diversification method or measure argues that Fund $\varepsilon$ is the best or most diversified. The up-side of the results not being similar is that if the results were identical, the PDI measure would have no use, as it provides the same results as a method that has already been tried, tested and proven to work.

Thus far the analysis cannot provide conclusive proof that the PDI is a good or better diversification measure compared to the traditional diversification measure therefore different performance measures will be introduced to help achieve the study's objective. The following section introduces concentration.

\subsection{Concentration results}

TABLE 3 shows that Fund $C$ is the most concentrated of all the funds, while Fund $\varepsilon$ is the least 
concentrated. Smith (2006) states that there is a statistical significant relationship between concentration and diversification and that this relationship is an inverse one. Thus, as the relationship is of an inverse nature the level of diversification will decrease as the concentration level increases and vice versa. The concentration results from TABL 3 thus state (in theory) that Fund $\varepsilon$ is the most diversified, while Fund $C$ has the lowest degree of diversification, as an increase in concentration (in theory) increases risk and vice versa. Also note that the JSE All Share Index (ALSI), which acts as the market index for the regression analysis, has a concentration index of $5.18 \%$, while Smith (2006) found the ALSI (market capitalisation weighted) to have a concentration of $5.35 \%$.

TABLE 3: Fund concentration and PDI results

\begin{tabular}{cccc}
\hline Fund & PDI & Concentration (HHI) & Res Var \\
\hline A & 4.44 & $4.21 \%$ & $5.55 \varepsilon-04$ \\
B & 7.68 & $3.71 \%$ & $5.84 \varepsilon-02$ \\
C & 1.02 & $5.22 \%$ & $3.16 \varepsilon-03$ \\
D & 12.7 & $2.88 \%$ & $4.90 \varepsilon-04$ \\
E & 6.51 & $2.25 \%$ & $4.61 \varepsilon-04$ \\
\hline
\end{tabular}

Source: Statistical analysis

Comparing the concentration results with the results of the diversification measures, PDI and residual variance, the fund which is most concentrated should be the fund which is least diversified in theory and vice versa. The comparison of the concentration and the diversification results, however, does not provide concrete conclusions about the effectiveness of the two diversification measures. Furthermore, the question remains whether concentration is the most appropriate method to distinguish between the two diversification methods. Another question is whether or not the PDI agrees with the inverse relationship theory.

\subsection{Relationship between PDI and concentration}

It is evident from the OLS regression analysis (PDI versus HHI) performed in Eviews $($ (2008) that the PDI is in line with the expected theory of an inverse relationship between diversification and concentration. The regression results thus establish that as the concentration measure ( $\mathrm{HHI})$ increases, the level of diversification (PDI score) decreases. The output of the regression analysis indicates that $53 \%$ of fund diversification (dependent variable) is explained or influenced by fund concentration (independent variable). TABLE 4 presents the regression results, and even though the results are not statistically reliable due to being based on five observations, the results are nevertheless included, since the results are not the main focus of the paper. Also the regression results provide some preliminary encouraging evidence as well pointing the way for some potential future confirmatory work. 
TABLE 4: Regression results

\begin{tabular}{|c|c|}
\hline \multicolumn{2}{|c|}{ Regression results } \\
\hline Estimating equation & $y=-271.52+16.39 x$ \\
\hline No. of variables (n) & 5 \\
\hline$t$-stat & -1.84 \\
\hline$F$-value & 3.38 \\
\hline$p$-value & 0.16 \\
\hline $\mathrm{R}^{2}$ & 0.53 \\
\hline Adjusted $\mathrm{R}^{2}$ & 0.37 \\
\hline
\end{tabular}

Source: Statistical analysis

\subsection{Comparative results with performance measures}

The performance measures or ratios included measure different concepts, for example the Sharpe ratio measures the reward per unit of risk while the Treynor measure measures the portfolio's risk premium per unit of risk. This entails that the different performance measures are not directly comparable, while these performance measures also do not measure diversification, thus they cannot be directly compared to diversification measures like the PDI. The central idea of comparison, however, is that performance measures generally measure risk while a large amount of risk might be taken as a lack of diversification. This analysis, however, strives to present the results in the best manner possible in order to achieve the objective of this study.

Botha (2006) found that the Omega is a superior measure to both the Sharpe and Sortino ratios, and Kaplan and Knowles (2004) are furthermore of the opinion that the Omega ratio or statistic can be used as an alternative to the Sharpe ratio as a risk-adjusted performance measure. In comparison to the Sharpe ratio, which is influenced only by the first two moments, the Omega ratio takes into account all the moments of the distribution (mean, volatility, skewness, kurtosis and higher moments). This characteristic makes the 0 mega ratio especially adaptive to asymmetric distributions where risk is not captured solely by the volatility. The Omega divides returns into both losses and gains above and below a return threshold, and ultimately determines the probability-weighted ratio of returns above and below this threshold (Botha, 2006). In short, for a given threshold or target level of return ( $r$ ) the 0 mega ratio is the weighted gain/loss ratio relative to $r$ (Wilson, 2007). The Omega ratio is unlike most performance measures or ratios, as the 0mega expresses gains to losses rather than in the form of (expected) return/risk. A higher 0 mega ratio is more desirable than a low ratio; a high 0 mega means that there is more density return on the right of the threshold return than on the left side. For detailed reading on the Omega ratio refer to Shadwick and Keating (2002) and Keating and Shadwick (2002).

As this study is concerned with diversification and thus risk, the focus must be on the downside in the Omega function (left of the $x$-axis), as this is mainly where risk is manifested. The ideal is for the Omega slope of a fund to be as steep as possible (in the downside) as this indicates few(er) negative outliners (returns). It is evident from FIGURE 3 that Fund C's slope is not very steep compared to the other funds and thus has more outliers or negative returns in the downside than the other funds. This is not good, as it increases the risk of the fund. Funds $\varepsilon$ and 
D perform the best in the downside. According to the Omega these two funds have the steepest slopes and are thus the least risky of the funds compared to the other funds.

In order to rank the funds in terms of risk and thus ultimately to be able to compare the ranking results, a threshold or point of comparison needs to be established. For this a randomly selected negative return (value) which falls in the downside $(-2 \%)$ is used, because firstly risk for any investor is manifested in negative returns and secondly because the 0 mega function is effective in judging risk by examining the downside. From FIGURE 3, which presents the 0 mega results at $2 \%$, it is clear that Fund $\varepsilon$ is the least risky, closely followed by Fund $D$, while Fund $C$ is the most risky.

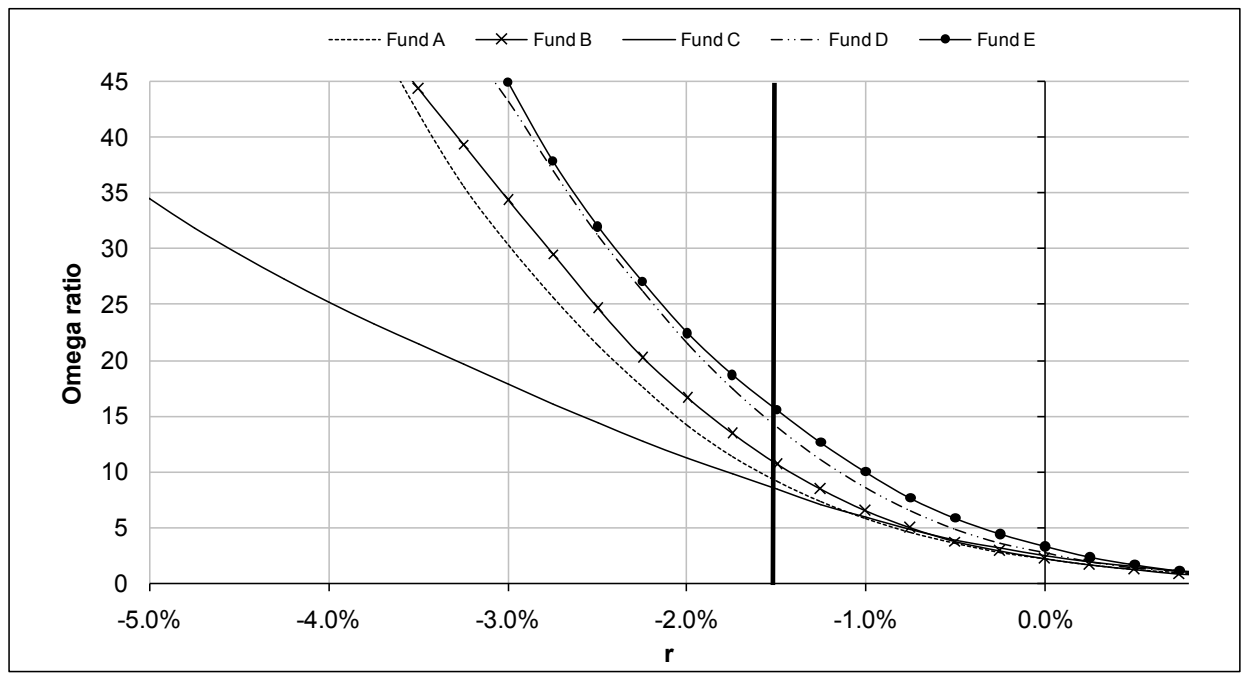

FIGURE 3: Omega at threshold $=-\mathbf{2} \%$

Source: Authors

TABLE 5 presents the comparative ranking results of the 0mega and the two diversification measures. It is clear from TABLE $\mathbf{5}$ that the PDI ranking results are very much in line with the ranking results of the 0 mega, especially if Fund $\varepsilon$ is omitted.

\section{TABLE 5: Comparative ranking results (0mega)}

\begin{tabular}{cccc} 
& & & RANKING \\
& PDI & Omega & Residual Variance \\
\hline Best & D & $\varepsilon$ & $\varepsilon$ \\
& B & D & D \\
& $E$ & B & A \\
& A & A & $\mathrm{C}$ \\
\hline
\end{tabular}

Source: Compiled by authors

TABLE 6 presents a summary of the fund ranking results, from which it can be concluded that neither of the two diversification measures is $100 \%$ in line with all the performance measures. As 
it is often recommended that the Sharpe and Sortino ratios should be considered alongside each other, these two measures were also combined. The combining process is done by averaging the four year results of each fund's Sharpe and Sortino ratio.

TABLE 6: Summary of results

\begin{tabular}{|c|c|c|c|c|c|}
\hline Ranking key & Best & & $\Rightarrow$ & & Worst \\
\hline PDI & $\mathrm{D}$ & $B$ & $\varepsilon$ & $A$ & C \\
\hline RV & $\varepsilon$ & $\mathrm{D}$ & A & C & B \\
\hline Concentration & $\varepsilon$ & $\mathrm{D}$ & B & A & C \\
\hline Std Deviation & A & B & $D$ & $\varepsilon$ & C \\
\hline Sharpe & $\varepsilon$ & $\mathrm{D}$ & C & $A$ & B \\
\hline Sortino & $\varepsilon$ & C & $D$ & B & A \\
\hline Sharpe and Sortino Combined & $\varepsilon$ & C & $D$ & B & A \\
\hline Treynor & D & C & A & $\varepsilon$ & B \\
\hline Omega at -2 threshold & $\varepsilon$ & $\mathrm{D}$ & B & $A$ & C \\
\hline Beta & D & $B$ & $\varepsilon$ & $A$ & C \\
\hline Information Ratio & $\varepsilon$ & $A$ & $D$ & B & C \\
\hline
\end{tabular}

Source: Compiled by authors

There was no consistent fund ranking from either the PDI or the RV method with any of the performance measures. It is clear, however, that the fund ranking results of the PDI and the 0 mega are the results most aligned, especially when Fund $\varepsilon$ is omitted from the results. The comparative 0 mega results will briefly be presented in the following section, as the 0 mega is the performance measure which is 'most' aligned with the PDI. Furthermore, as the Omega is argued to be the best measure of the measures used in the empirical study in terms of what it measures and especially how it measures, Omega is used to attain this study's second objective of determining whether the PDI can be used as a tool by fund managers.

\section{RESULTS - PDI AS A FUND MANAGEMENT TOOL}

\subsection{PDI as a fund management tool}

A scenario is created to ascertain whether the PDI can be used as a (alternative) fund management tool when constructing or customising (changing the composition) of an existing portfolio. The aim is to use the PDI as a tool to improve a fund's diversification, i.e. reduce the risk of the fund without a concomitant reduction in return.

From FIGURE 3 it can be observed that Fund $C$ is far below the other funds in the downside, while Fund C's slope is also not nearly as steep as the slopes of the other funds. Fund C thus has more risk than the other funds, according to Omega. The PDI measure shares this sentiment, as Fund C has the lowest PDI score of all the funds analysed, namely 1.02. In order to reduce the amount of risk in Fund $C$, which is the objective of diversification, the (Omega) slope of Fund $C$ must be improved specifically in the downside. The results obtained thus far show that the PDI of Fund $C$ will also improve as the (Omega) slope of Fund C becomes steeper (improves). 


\subsection{Fund C optimisation}

As it is the goal of Modern Portfolio Theory to find a balance between risk and return in order to ultimately achieve the optimal portfolio, Fund $C$ needs to be optimised (by weight-adjusting each share in the portfolio in order to obtain the minimum portfolio variance for a given level of return). The PDI is used to identify which shares in Fund $C$ should receive more weighting and which shares should receive less weighting before Fund $C$ is optimised (in order for the PDI to be considered a tool) using an optimisation model. If the shares in Fund $\mathrm{C}$ are weighted as suggested by the PDI, i.e. the truly independent components or shares receiving more weight, Fund $\mathrm{C}$ should be more diversified, given that the same shares are contained in Fund $\mathrm{C}$.

The process of using the PDI as a tool in order to reduce Fund C's risk is as follows:

- optimise Fund C using the information obtained from the PDI results. The optimisation model will provide the optimal weight for each share in Fund $C$,

- calculate the weekly returns of Fund C with the 'new' (optimal) weights obtained from the optimisation model,

- calculate the Omega with the 'new' weekly returns, and

- calculate the PDI with the 'new' weekly returns.

The period of analysis again ranges from 1 July 2003 to 30 June 2007, and the shares contained in Fund $C$ remain similar to those in the original portfolio. The RFR will be taken as the four-year average RFR ( $8.12 \%$ ), as was done throughout this study, while the level of return (required rate of return) that is used for optimisation is the RFR $+1 \%(9.12 \%)$, as this is only for illustration purposes where the process and concept is the primary focus. 2000 iterations were run on the optimisation model for all optimisation procedures.

The minimum weight constraint of each share in Fund $\mathrm{C}$ is $0 \%$ except for the seven independent shares as identified by the PDI for which the minimum constraint is $3.89 \%$. This minimum constraint is estimated by optimising Fund $C$ without any constraints and then using the average optimal weight of the independent shares as the minimum constraint. The maximum weight constraint of each share in Fund C is $100 \%$, except for the three non-independent shares, in relation to which it is $5.28 \%$. This is estimated using the same procedure as that used for acquiring the minimum constraint, except that the procedure is performed on the nonindependent shares.

\subsection{Results of PDI as a fund management tool}

TABLE 7 presents the results of Fund C before and after optimisation with the aid of the PDI.

TABLE 7: Comparative results

\begin{tabular}{lcc}
\hline & Pre-optimisation & Post-optimisation \\
\hline PDI & 1.02 & 2.25 \\
$\mathrm{HHI}$ & $5.22 \%$ & $5.17 \%$ \\
\hline
\end{tabular}

Source: Compiled by authors

The increase of Fund C's PDI (score) is an indication that Fund C's degree of diversification 
increased, as a larger PDI is preferred and indicates a larger degree of diversification than a small PDI score. The PDI score of Fund C after optimisation (2.25) indicates that Fund $C$ is far from completely diversified, as a PDI score of 34 would be the fund's PDI score if the fund was completely diversified (Fund C contains 34 shares). However, Fund $C$ is more diversified than before the fund was optimised and the most independent factors or shares identified. Note that Fund C's HHI has declined to $5.17 \%$ from $5.22 \%$ before Fund C was optimised with the help of the $\mathrm{PDI}$. The decrease in the concentration of Fund $\mathrm{C}$ is also in line with the inverse relationship between diversification and concentration according to theory.

FIGURE 4 presents the Omega results before and after Fund $C$ is optimised with the assistance of the PDI in identifying the most independent and non-independent shares of Fund C. FIGURE $\mathbf{5}$ illustrates Fund C's optimisation results along with the Omega functions of the other funds, where $-2 \%$ is also used as an evaluation (comparison) point.

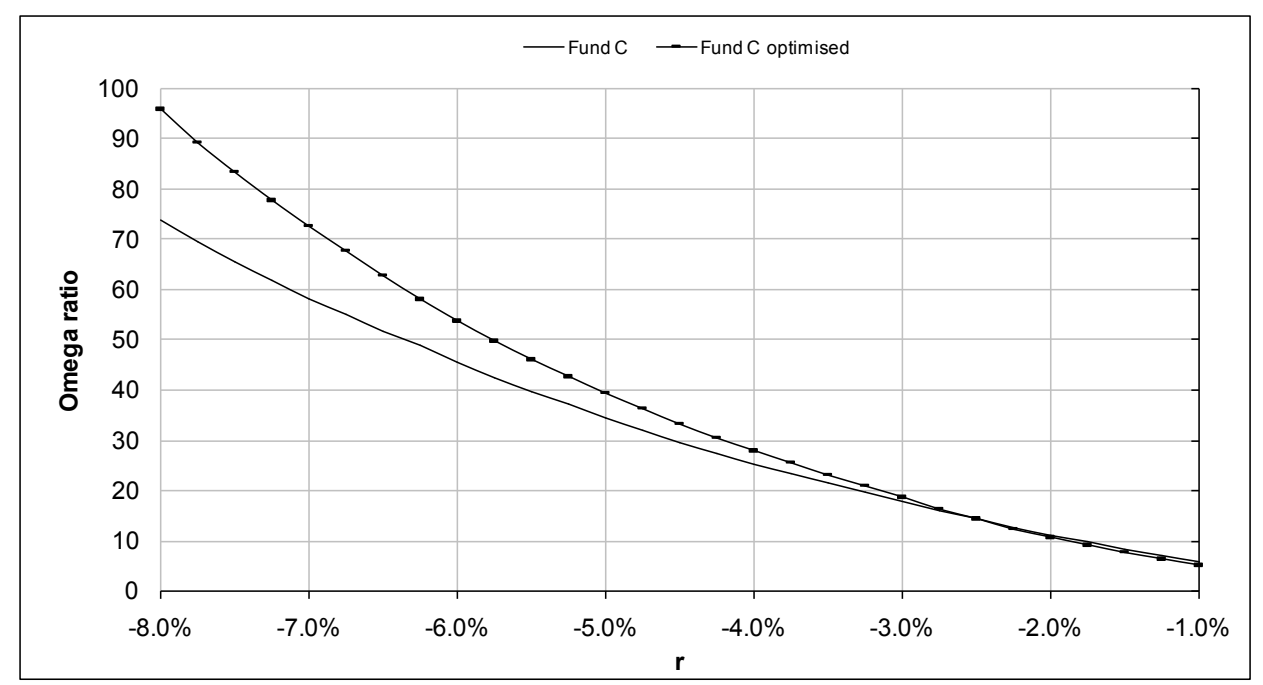

\section{FIGURE 4: Fund C's optimised Omega results}

\section{Source: Compiled by authors}

In FIGURE $\mathbf{4}$ it is clear that the optimisation of Fund $\mathrm{C}$ made a difference in the downside as the slope of Fund $C$ improved (became steeper). Fund $C$ is thus clearly less risky after it has been optimised with the assistance of the PDI.

In FIGURE $\mathbf{5}$ it is clear that the optimisation of Fund $\mathrm{C}$ made a difference in the downside, especially below $-2 \%$. It would have been ideal if the slope of the optimised Fund $C$ became steeper at a faster rate, thus earlier in the downside. The result, however, is still an improvement on Fund $\mathrm{C}$ before the optimisation process, which was aided by the PDI. As the slope of Fund $C$ is steeper after optimisation, Fund $C$ is less risky, and this can be attributed to an increase of diversification as the PDI (score) of Fund C also increased. 


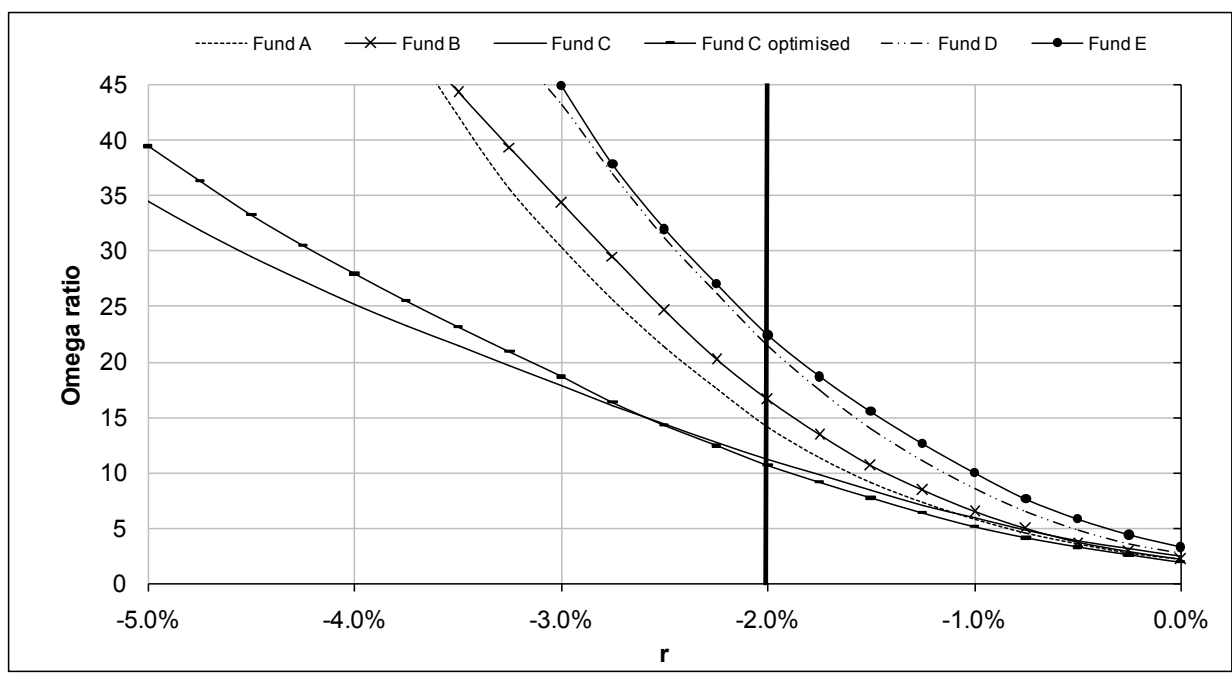

FIGURE 5: Fund C's optimised Omega results at $-2 \%$

\section{Source: Compiled by authors}

\subsection{Marginal portfolio diversification}

Marginal portfolio diversification (marginal PDI) refers to the additional diversification effect an additional security or share has on the portfolio, i.e. marginal portfolio diversification aims to determine how much additional diversification is gained by adding additional share(s) to the portfolio by utilising the PDI. Equation (3) can be used to calculate the marginal PDI. A new portfolio or fund, Fund $Z$, is constructed in order to illustrate marginal portfolio diversification with the use of the PDI. Fund $Z$ contains 10 randomly selected shares from the Johannesburg Securities Exchange (JSE), which were also chosen from the entire universe of funds used in the first empirical study. The period for calculation is 1 July 2003 to 30 June 2007, again using weekly closing prices and returns. Each share is assigned a weight of $10 \%$ of the portfolio to constitute an equally weighted portfolio.

The data required in order to calculate the marginal PDI is the periodic returns of the 10 shares which constitute Fund $Z$.

The procedure to calculate the PDI for Fund Z:

- a column consisting of the time series returns of shares, which make up Fund $Z$, multiplied by the respective weight of the $\mathrm{N}$ shares in Fund $\mathrm{Z}$

- a Principal Component Analysis (PCA) is then conducted on a covariance matrix of these series (a series of uncorrelated factors (eigenvalues) that represent the volatility of the returns is the result of the PCA)

- these factors are then ordered from most to least significant and lastly

- these factors are substituted into the PDI formula (equation (3)). 


\subsection{Marginal PDI results}

Fund $Z$ has a PDI score of 4.09 before any alterations or changes. When the MTN share is removed from Fund Z, however, the PDI score of the fund declines to 3.63. Thus it can be concluded that the MTN share adds or contributes 0.46 (4.09 minus 3.63) in terms of diversification to Fund Z. This PDI score of 0.46 is equal to a significant $11.25 \%$ positive diversification contribution to Fund $Z$. It should be remembered, however, that the diversification 'value' that a specific share, in this case MTN, provides to a fund will differ according to the shares included in the fund, and how these shares correlate, as well as the weighting structure of the fund. When the Sasol share is omitted from Fund Z the PDI score of the fund declines to 3.68 (MTN share again included in Fund $Z$ ). Thus by subtracting the PDI score of Fund Z, when the Sasol share was omitted from the original Fund Z, we can conclude that the Sasol share adds 0.41 to Fund $Z$ in terms of diversification. The Sasol share's PDI score of 0.41 constitutes a $10.02 \%$ (positive) diversification contribution to Fund $Z$, which again is a significant amount of diversification benefit added to the fund by one share. FIGURE 6 presents the marginal PDI results as evaluated by the 0 mega.

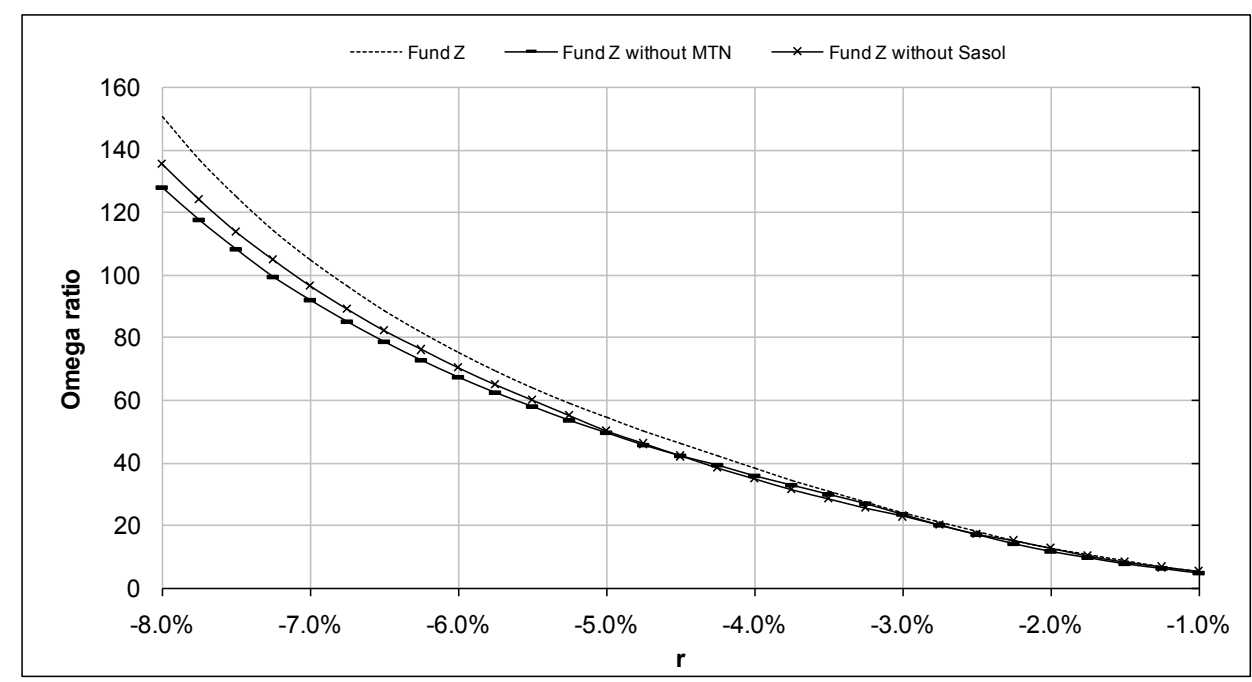

FIGURE 6: Marginal PDI

Source: Compiled by authors

From FIGURE $\mathbf{6}$ is can be seen that the omission of both the MTN and Sasol shares from Fund Z decreases the diversification of Fund Z, as the Omega slope of Fund Z decreases in steepness when these shares are omitted from Fund $Z$. It is thus clear that the Omega results are in line with the PDI results.

\subsection{Return and diversification matrix}

If the historic return performance and the PDI (score) of each of the shares or potential shares of a fund are known the fund manager can present them as in FIGURE 7, which indicates return versus diversification benefits per share (1 June 2003 to 30 June 2007). 
A fund manager would prefer shares that plot as high and a far right as possible (such as FIGURE 7), with share return performance represented on the $y$-axis and share diversification benefit represented on the $x$-axis.

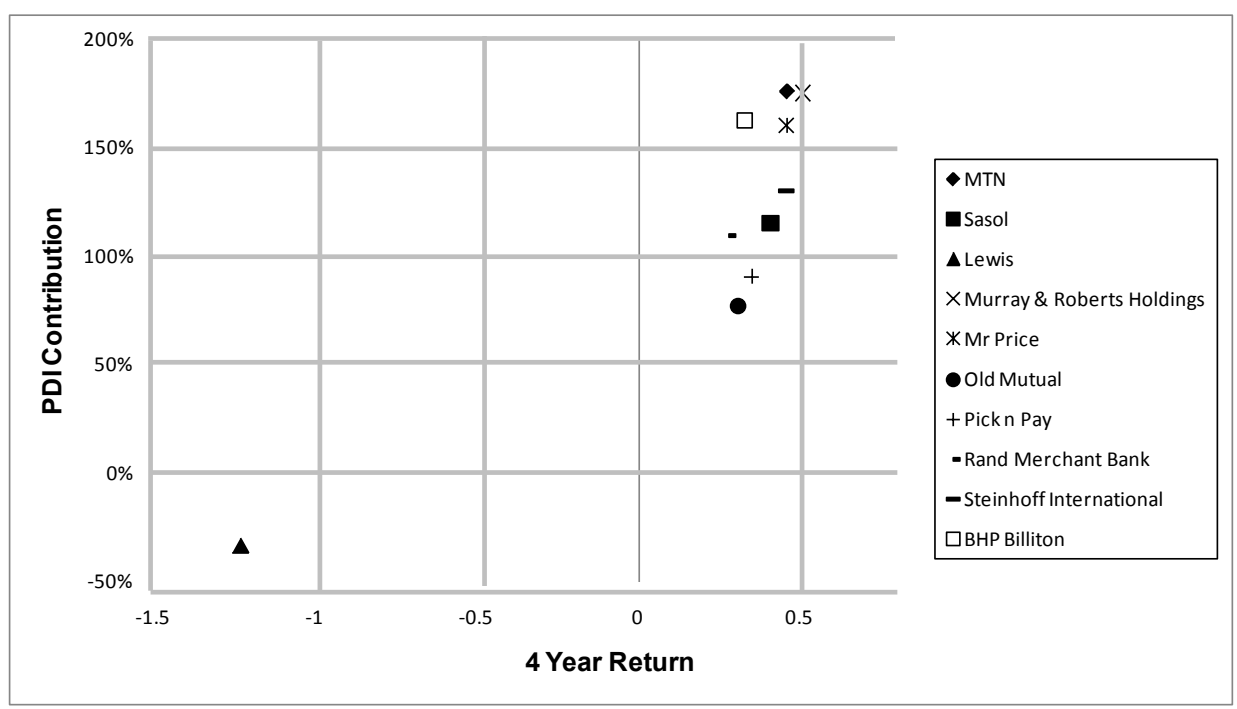

FIGURE 7: 4-year annual returns versus PDI contribution

\section{Source: Compiled by authors}

From FIGURE 7 the fund manager or investor can also establish an efficient frontier, which will yield a fund consisting of shares that will provide the highest degree of diversification for a certain level of return.

\section{CONCLUSION}

Neither of the two diversification measures delivered similar results to any of the risk-adjusted performance measures on a consistent basis, although the ranking results of the PDI and the Omega were very similar (although not aligned $100 \%$ ). In terms of the first objective of this study of whether or not the PDI is a good diversification measure compared to the RV method, it can be concluded that:

- the PDI may be a good measure of diversification compared to the RV method, although this is uncertain as no statistical analysis was used. The inclusion of a third diversification measure may provide more insight into which of the PDI or RV methods are more appropriate measures of diversification,

- the PDI has proven to a certain degree to be an alternative measure of diversification, as it delivers results which are different from the results obtained by the RV method, and

- the PDI may indeed be the superior diversification measure when a market is of a highly concentrated nature, as the PDI is free from market influence.

Secondly, it can be concluded that the PDI can be used as a tool by a portfolio manager when 
constructing or customising (changing the composition of) an existing portfolio, i.e. reduce the risk of a portfolio without a concomitant reduction in return. This can be concluded as the Omega results confirm the results obtained by the PDI in this, the second, empirical study. Furthermore, the PDI proved that it can be successfully used in a marginal portfolio diversification concept to ascertain the degree of diversification that a certain share or security provides to an existing portfolio.

Finally, the PDI and the Omega ratio can be used in conjunction or as substitutes, as these two measures are correctly aligned, while these measures can also be used to evaluate the results of one another. The PDI might thus offer portfolio managers the opportunity to flex (the use of) the PDI to a certain degree, but how far the PDI can be flexed remains to be seen. Ultimately the PDI is a useful measure of diversification, and might prove useful to the BCBS when concentration and or diversification measures are once again considered in future.

\section{FUTURE RESEARCH SUGGESTIONS}

As the PDI is a new measure, it is expected that the amount of research concerning the PDI will increase as the measure gains acceptability in the market. Suggestions for future research which are more specific to this study include a similar comparison methodology with the inclusion of a third diversification measure in order to ascertain which (the PDI or RV) is the more 'correct' or better measure of diversification. The inclusion of out-of-sample testing might also provide added assurance in the results obtained. Another possibility might be similar research which focuses on the active management setting. Lastly, possible future research might be to repeat this study with the incorporation of a statistical method for empirical testing instead of the ranking results method used in this study.

\section{LIST OF REFERENCES}

Barnea, A. \& Logue, D.E. (1973). Stock market based measures of corporate diversification. Journal of Industrial Economics, 22(1), pp. 51-60.

Botha, M. (2006). A comparison of South African hedge fund performance measures. South African Journal of Economics, 75(3), pp. 459-477.

Correia, C., Flynn, D., Uliana, $\varepsilon$. and Wormald, M. (2003). Financial management, $5^{\text {th }}$ edition. Cape Town: Juta.

D’Ambrosio, C.A. (1976). Principles of modern investments. Chicago: Science Research Associates.

Equinox. (1999). Fund performances. [On-line] Available:

http://www.equinox.co.za/unittrusts/funds/fund Performances.asp. (Accessed 12 March 2007)

Eviews $($ C. (2008). Eviews 6 overview. [0n-line] Available:

http://www.eviews.com/eviews6/eviews6/over view/ev6over.html. (Accessed 30 June 2008)

Fung, W. \& Hsieh, D.A. (1997a). Empirical characteristics of dynamic trading strategies: the case of hedge funds. The Review of Financial Studies, 10(2), pp. 275-302.

Fung, W. \& Hsieh, D.A. (1997b). Survivorship bias and investment style in the returns of CTAs. The Journal of Portfolio Management, 24(1), pp. 30-41. 
Fung, W. \& Hsieh, D.A. (2002). Asset-based style factors for hedge funds. Financial Analysts Journal, 58(5), 16-27.

Gopi, Y., Dugmore, B. \& Bradfield, D. (2006). A new measure of portfolio diversification. Unpublished. Hattingh, J.J. (2006). Portfolio management: The use of alternative investments for the purpose of diversification. Dissertation - M.Com. Johannesburg: RAU.

Hovenkamp, H. (1993). Antitrust, $2^{\text {nd }}$ edition. St. Paul, MN: West Publishing.

Jolliffe, I.T. (1986). Principal Component Analysis. New York: Springer-Verlag.

Jolliffe, I.T. (2002). Principal Component Analysis, $2^{\text {nd }}$ edition. New York: Springer-Verlag.

Keating, C. \& Shadwick, W. (2002). An introduction to Omega. London: The Finance Development Centre. [On-line] Available: http://finance.yendor.com/etfviz/2007/0928/0mega-intro.pdf. (Accessed 27 July 2007)

Kaplan, P.D. \& Knowles, J.A. (2004). Kappa: A generalised downside risk-adjusted performance measure. [On-line] Available: http://www.edge-fund.com/KaKn04.pdf. (Accessed 27 July 2007)

Markowitz, H.M. (1952). Portfolio selection. Journal of Finance, 7(1), pp. 77-91.

Reilly, F.K. \& Brown, K.C. (2006). Investment analysis and portfolio management, $8^{\text {th }}$ edition. Mason, $\mathrm{OH}$ : Thompson South-Western.

Rencher, A.C. (1998). Multivariate statistical inference and applications. Canada: Wiley.

Rudin, A.M. \& Morgan, J.S. (2006). A Portfolio Diversification Index. Journal of portfolio management, 32(2), pp. 81-89.

SAS ${ }^{\circledR}$. (2009). Products and Solutions/Statistical Analysis. [0n-line] Available: http://www.sas.com/technologies/analytics/statistics/stat/index.html. (Accessed 30 June 2008)

Shadwick, W. \& Keating, C. (2002). A universal performance measure. Journal of Performance Measurement, 6(3), pp. 59-87.

Smith, D. (2006). A portfolio diversification index as a fund management instrument. (ReportMBA). Cape Town: UCT.

Stallman, C. (2004). Diversification vs. concentration. [0n-line] Available: http://www.teenanalyst.com/general/diversification.html. (Accessed 2 June 2008)

Statman, M. (1987). How many stocks make a diversified portfolio? Journal of financial and quantitative analytics, 22(3), pp. 353-363.

Wilson, R. (Richard@HedgeFundGroup.org) (2007).Omega Ratio:Omega ratio explanation. Discussion group. [0n-line] Available: http://richard-wilson.blogspot.com/2007/10/omegaratio.html. (Accessed 27 July 2007) 
IMPROVED INVESTMENT PERFORMANCE USING THE PORTFOLIO DIVERSIFICATION INDEX 